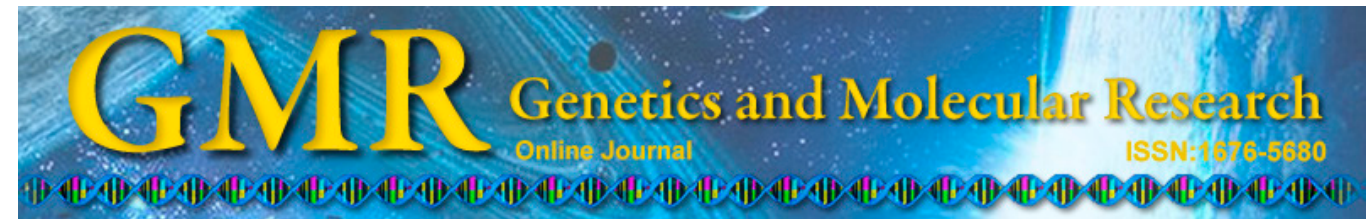

\title{
Nuclear and mitochondrial genome instability induced by senna (Cassia angustifolia Vahl.) aqueous extract in Saccharomyces cerevisiae strains
}

\author{
C.R. Silva ${ }^{1,2,3}$, A. Caldeira-de-Araújo ${ }^{1}$, A.C. Leitão ${ }^{2}$ and M. Pádula ${ }^{2,3}$ \\ 'Laboratório de Radio e Fotobiologia, Departamento de Biofísica e Biometria, \\ IBRAG, Universidade do Estado do Rio de Janeiro, Rio de Janeiro, RJ, Brasil \\ ${ }^{2}$ Laboratório de Radiobiologia Molecular, \\ Universidade Federal do Rio de Janeiro, Rio de Janeiro, RJ, Brasil \\ ${ }^{3}$ Laboratório de Microbiologia e Avaliação Genotóxica, \\ Faculdade de Farmácia, Centro de Ciências da Saúde (A2-42), \\ Universidade Federal do Rio de Janeiro UFRJ, Rio de Janeiro, RJ, Brasil \\ Corresponding author: M. de Pádula \\ E-mail: marcelo@pharma.ufrj.br
}

Genet. Mol. Res. 13 (4): 9861-9866 (2014)

Received February 17, 2014

Accepted May 29, 2014

Published November 27, 2014

DOI http://dx.doi.org/10.4238/2014.November.27.13

\begin{abstract}
Cassia angustifolia Vahl. (senna) is commonly used in selfmedication and is frequently used to treat intestine constipation. A previous study involving bacteria and plasmid DNA suggested the possible toxicity of the aqueous extract of senna (SAE). The aim of this study was to extend the knowledge concerning SAE genotoxicity mechanisms because of its widespread use and its risks to human health. We investigated the impact of SAE on nuclear DNA and on the stability of mitochondrial DNA in Saccharomyces cerevisiae (wt, ogg1, msh6, and ogg1msh6) strains, monitoring the formation of petite mutants. Our results demonstrated that SAE specifically increased $\mathrm{Can}^{\mathrm{R}}$ mutagenesis only in the msh6 mutant, supporting the view that SAE can induce misincorporation errors in DNA.
\end{abstract}


We observed a significant increase in the frequency of petite colonies in all studied strains. Our data indicate that SAE has genotoxic activity towards both mitochondrial and nuclear DNA.

Key words: DNA repair; Genome instability; Oxidative damage; petite colonies; Saccharomyces cerevisiae

\section{INTRODUCTION}

Many herbal laxatives, such as Cassia angustifolia Vahl. (senna), have been used for self-medication in the treatment of intestinal constipation (Brusick and Mengs, 1997; Van Gorkon et al., 1999).

In our previous study, we assessed the possible toxicity of senna. We reported that the aqueous extract of senna (SAE) exhibited highly genotoxic activity such as DNA strand breaks against plasmid DNA in a cell-free system (Silva et al., 2008). However, SAE showed antioxidant/antimutagenic activity in Escherichia coli cells upon $\mathrm{H}_{2} \mathrm{O}_{2}$ treatment. This feature of SAE was observed in the E. coli IC203 (uvrA oxyR) and IC205 (uvrA mutM) strains, which are highly sensitive to oxidative mutagens (Silva et al., 2008).

As part of a continuous effort to understand the toxic effects of senna, we examined SAE genotoxicity mechanisms in Saccharomyces cerevisiae.

S. cerevisiae is an interesting eukaryotic model for genetic studies, as a number of yeast proteins have been shown to be functionally interchangeable with human proteins (Jazayeri and Jackson, 2002; Mager and Winderickx, 2005). Additionally, yeast are useful for understanding base excision repair, genetic recombination, and response to stresses involved in cellular and organism aging (Gershon and Gershon, 2000; Prakash and Prakash, 2000; Rowe et al., 2008).

Oxidative damage produced by intracellular reactive oxygen species results in DNA lesions, as base modifications, and in the formation of apurinic/apyrimidinic lesions, which may be toxic and/or mutagenic. Mutagenic 8-oxo-7, 8-dihydroguanine (8-oxoG) lesions are present in elevated levels in aged and cancerous cells (Rowe et al., 2008).

In $S$. cerevisiae, mismatch repair (MMR) and translesion synthesis cooperate with Ogg1 to prevent the mutagenic effect of 8-oxoG in nuclear DNA (Girard and Boiteux, 1997; Boiteux et al., 2002; de Pádula et al., 2004). Msh2/Msh6 proteins contribute to the prevention of GC to TA tranversions in nuclei, as the Msh6 protein can remove adenine from 8-oxoG:A pairs (Boiteux et al., 2002). In addition, Ogg1 has been implicated in the prevention of petite mutants of S. cerevisiae (Singh et al., 2001), indicating a role of Ogg1 in avoiding 8-oxoG in mtDNA. The Msh1 protein has been implicated in mitochondrial stability, indicating that MMR occurs in mitochondria (Larsen et al., 2005).

In this study, we evaluated the genotoxic potential of SAE using a series of S. cerevisiae strains in 2 experimental assays, including determination of canavanine-resistant mutants and determination of mitochondrial mutants (petite colonies) upon SAE treatment.

\section{MATERIAL AND METHODS}

\section{Phytopharmaceutical, chemical agents, and reagents}

Orient Mix Fitoterápicos do Brasil, Ltd. (Rio de Janeiro, Brazil) provided powdered 
senna leaves in capsules; each capsule contained 35\% sennosides, A and B, according to data from bulla. The $\mathrm{SAE}$ in $0.9 \% \mathrm{NaCl}$ sterile solution was extemporaneously prepared for each experiment (Silva et al., 2008). Bacto agar, bacto-peptone, yeast nitrogen base without amino acids, and bacto yeast extract were purchased from Difco Laboratories (Detroit, MI, USA). Sodium chloride, potassium dihydrogen phosphate, sodium phosphate dibasic dodecahydrate, triphenyltetrazolium chloride, sodium bicarbonate sodium hydroxide, and glycose were obtained from Merck (White House Station, NJ, USA); uracil, L-lysine, L-tryptophan, L-leucine, L-canavanine sulfate salt, and L-histidine monohydrochloride monohydrate were from Sigma-Aldrich (St. Louis, MO, USA). Ultra-pure water was obtained using a Milli-Q water system from Millipore Corporation (Billerica, MA, USA).

\section{Media and growth conditions}

S. cerevisiae strains FF18733 (MATa, leu2-3-112, trp1-289, his7-2, ura3-52, lys1-1), CD138 (as FF18733 but ogg1 $:: T R P 1$ ), BPS1031(as FF18733 but msh64::kANMX6), and

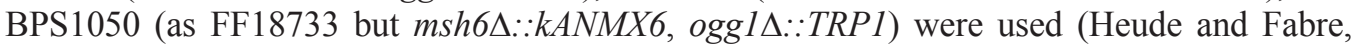
1993; Thomas et al., 1997; Melo et al., 2004; de Pádula et al., 2004). Cells were grown at $30^{\circ} \mathrm{C}$ in YPD medium ( $1 \%$ yeast extract, $1 \%$ bacto-peptone, $2 \%$ glucose, with $2 \%$ agar for plates) or yeast nitrogen base dextrose (YNBD) medium ( $0.7 \%$ YNBD without amino acids, $2 \%$ glucose, with $2 \%$ agar for plates) supplemented with appropriate amino acids and bases (de Pádula et al., 2004). Supplemented YNBD medium lacking arginine but containing canavanine (60 mg/L) was used for the selective growth of canavanine-resistant $\left(\mathrm{Can}^{\mathrm{R}}\right)$ mutants (de Pádula et al., 2004).

\section{Spontaneous and induced mutation frequencies}

Yeast strains were grown in $2 \mathrm{~mL}$ YPD medium with for 2 days at $30^{\circ} \mathrm{C}$. For each strain, 10 independent cultures in YPD $(2.0 \mathrm{~mL})$ were inoculated with approximately $2 \times 10^{2}$ cells and grown at $30^{\circ} \mathrm{C}$ for 2 days with $\mathrm{SAE}$ or $0.9 \% \mathrm{NaCl}$ sterile solution. A concentration of $500 \mu \mathrm{g} / \mathrm{mL}$ SAE was chosen for these experiments based on our previous study in which this concentration was generated DNA strand breaks in plasmid DNA (Silva et al., 2008). Cell density was measured by plating dilutions on YPD agar plates and counting the colonies after 2-3 days of growth at $30^{\circ} \mathrm{C}$. The quantification of $\mathrm{Can}^{\mathrm{R}}$ mutants was determined after plating on selective medium (YNBD-canavanine). All experiments were carried out independently 3 times (Melo et al., 2004). Mutation frequencies were determined as $\mathrm{Can}^{\mathrm{R}}$ per $10^{7}$ cells (Melo et al., 2004).

\section{Measurement of mitochondrial mutants}

Petite mutants form white colonies (petite colonies). Cultures were diluted, plated on YPD, and incubated at $30^{\circ} \mathrm{C}$ for 3-4 days. Spontaneous and induced mitochondrial mutants were scored using a triphenyltetrazolium chloride color assay (Ogur et al., 1957).

\section{Statistical analysis}

The results were analyzed by analysis of variance when i) the data were normally 
distributed as verified by the Kolmogorov and Smirnov method and ii) samples from the populations showed identical standard deviations as verified using the Bartlett method. Analysis of variance was followed by the Student-Newman-Keuls multiple comparison test using the statistical program InStat version 3.01 (GraphPad Software, San Diego, CA, USA). A significance level of $5 \%$ was used to evaluate the data.

\section{RESULTS}

SAE-induced mutagenesis in $S$. cerevisiae was assessed using FF18733 (wild-type, $w t$ ), CD138 (oggl), BPS1031 (msh6), and BPS1050 (msh6oggl) strains. SAE was able to specifically increase mutagenesis only in $m s h 6$ cells $(\mathrm{P}<0.05)$. In this strain, the number of $\mathrm{Can}^{\mathrm{R}}$ mutants $/ 10^{7}$ cells (699) was 6-fold higher than of the rate of spontaneous revertants (116) after $96 \mathrm{~h}$ of treatment with SAE, while no significant increase was observed in the other strains tested $(\mathrm{P}>0.05)$.

Regarding mitochondria mutagenic damage, SAE increased the number of respiratory mutants in all strains tested. The petite colonies fold-increase over spontaneous levels were $w t$ : 2.6; ogg 1: 3.3; msh6: 2.5, and msh6ogg1: 1.7 after $96 \mathrm{~h}$ of treatment with SAE. These results indicate a distinct genotoxicity pattern of SAE in eukaryotic cells, as nuclei and mitochondria endure different types of DNA damage.

\section{DISCUSSION}

In the present study, we investigated the impact of SAE on mutagenesis and mitochondrial stability in S. cerevisiae strains.

We evaluated whether SAE was genotoxic in S. cerevisiae by monitoring the ability of SAE to induce mutations in $w t, o g g 1, m s h 6$, and $\operatorname{ogg} 1 m s h 6$ strains.

Our results showed that SAE specifically increased $\mathrm{Can}^{\mathrm{R}}$ mutagenesis only in the msh6 mutant, supporting that SAE induces misincorporation errors in nuclear DNA, as MSH6 plays a direct role in MMR, eliminating pre-mutagenic base mismatches (Ni et al., 1999). As no increase in mutagenesis was observed in oggl mutants, the induction of 8-oxoG by SAE can be ruled out, as well as the formation of 8-oxoG:A mispairs in nuclear DNA. Notably, SAE did not increase the number of $\mathrm{Can}^{\mathrm{R}}$ mutants of the msh6oggl strain. In fact, double inactivation of OGG1 and MSH6 genes had a synergistic effect on spontaneous mutagenesis (Radak and Boldogh, 2010). This may compromise the detection of other base substitutions, as approximately $80 \%$ of base substitution events are spontaneous GC to TA transversions in this strain (Ni et al., 1999; Radak and Boldogh, 2010). These results reinforce the notion that SAE-induced DNA damage involves other pre-mutagenic mismatches, likely leading to base pair substitutions such as GC to AT, GC to TA, and AT to GC, independently of 8-oxoG generation (Boiteux et al., 2002).

In addition, we observed a significant increase in the frequency of petite colonies induced by SAE treatment $(\mathrm{P}<0.05)$. In the $w t$ strain, SAE induced a 2.6-fold increase in the number of petite colonies, while a 3.3-fold increase was observed for the ogg1 strain. This indicates that SAE induced distinct DNA damage profiles according to DNA localization. In the nucleus, SAE-induced DNA damage was a substrate for Msh6 (MMR) but not for Ogg1 protein. In contrast, in mitochondria, DNA damage required Ogg1 protein to prevent SAEinduced petite mutant colonies. 
Interestingly, the msh6 mutant displayed a lower level of spontaneous petite mutants compared to the $w t$ strain. Although the Msh6 protein has not been described in mitochondria, MMR (Msh1) has been previously reported to be a backup for mitochondrial base excision repair (Dzierzbicki et al., 2004). Msh6 may interfere with and modulate the generation of petite mutants. As Ogg1 is needed to avoid spontaneous and SAE-induced petite mutants, 8-oxoG appears to be generated in mitochondria. However, this appears to occur in the nucleotide pool rather than in the mtDNA. If 8-oxoG is produced in the nucleotide pool, it can be incorporated in DNA opposite to adenine and can constitute a substrate to Msh6 mismatch repair. Additionally, adenine is eliminated from the 8-oxoG:A mismatch and, after one replication round, may lead to a TA to GC transversion. This renders the $w t$ strain more prone to mitochondrial instability compared to an msh6 mutant. Indeed, the results obtained with the double-mutant ogg1msh6 reinforce this hypothesis. Although inactivation of MSH6 in an oggl background was not sufficient for reducing the overall level of spontaneous petite mutants, it was able to partially suppress SAE-induced petite mutants compared to the ogg1 strain. This reinforces that SAE can induce DNA damage to $S$. cerevisiae in a bimodal manner. While SAE induces nuclear DNA damage requiring mismatch repair but not Ogg1 protein, in mitochondria SAE induces oxidative damage and relies on Ogg1 to prevent petite mutants.

\section{CONCLUSION}

Our data indicate a differential genotoxic pattern of SAE in the mitochondria and nucleus regarding DNA damage production.

\section{Conflicts of interest}

The authors declare no conflicts of interest.

\section{ACKNOWLEDGMENTS}

The authors thank MSc. Marcia B.N. de Oliveira (UERJ), MSc Simone Simplicio (UERJ), MSc Paulo Thiago S. Santos (UERJ), BSc Rita de Cássia Albuquerque (UFRJ) and BSc Janine S.C. Rurr (UFRJ) for their technical assistance; Dr. Alicia V. Pinto (FIOCRUZ) for critical reading of this manuscript. Research supported by CNPq (Proj. Universal - Proc. \#474280/2007-1) and FAPERJ (APQ1 \#111566/2010) Brazilian agencies.

\section{REFERENCES}

Boiteux S, Gellon L and Guibourt N (2002). Repair of 8-oxoguanine in Saccharomyces cerevisiae: interplay of DNA repair and replication mechanisms. Free Radic. Biol. Med. 32: 1244-1253.

Brusick D and Mengs U (1997). Assessment of the genotoxic risk from laxative senna products. Environ. Mol. Mutagen. 29: 1-9.

de Pádula M, Slezak G, Auffret van Der KP and Boiteux S (2004). The post-replication repair RAD18 and RAD6 genes are involved in the prevention of spontaneous mutations caused by 7, 8-dihydro-8-oxoguanine in Saccharomyces cerevisiae. Nucleic Acids Res. 32: 5003-5010.

Dzierzbicki P, Koprowski P, Fikus MU, Malc E, et al. (2004). Repair of oxidative damage in mitochondrial DNA of Saccharomyces cerevisiae: involvement of the MSH1-dependent pathway. DNA Rep. 3: 403-411.

Gershon H and Gershon D (2000). The budding yeast, Saccharomyces cerevisiae, as a model for aging research: a critical review. Mech. Ageing Dev. 120: 1-22. 
Girard PM and Boiteux S (1997). Repair of oxidized DNA bases in the yeast Saccharomyces cerevisiae. Biochimie 79: 559-566.

Heude M and Fabre F (1993). a/alpha-control of DNA repair in the yeast Saccharomyces cerevisiae: genetic and physiological aspects. Genetics 133: 489-498.

Jazayeri A and Jackson SP (2002). Screening the yeast genome for new DNA-repair genes. Genome Biol. 3: reviews 1009.1-1009.5

Larsen NB, Rasmussen M and Rasmussen LJ (2005). Nuclear and mitochondrial DNA repair: similar pathways? Mitochondrion 5: 89-108.

Mager WH and Winderickx J (2005). Yeast as a model for medical and medicinal research. Trends Pharmacol. Sci. 26: 265-273.

Melo RG, Leitão AC and Padula M (2004). Role of OGG1 and NTG2 in the repair of oxidative DNA damage and mutagenesis induced by hydrogen peroxide in Saccharomyces cerevisiae: relationships with transition metals iron and copper. Yeast 21: 991-1003.

Ni TT, Marsischky GT and Kolodner RD (1999). MSH2 and MSH6 are required for removal of adenine misincorporated opposite 8-oxo-guanine in S. cerevisiae. Mol. Cell 4: 439-444.

Ogur M, St. John R and Nagai S (1957). Tetrazolium overlay technique for population studies of respiration deficiency in yeast. Science 125: 928-929.

Prakash S and Prakash L (2000). Nucleotide excision repair in yeast. Mutat. Res. 451: 13-24.

Radak Z and Boldogh I (2010). 8-Oxo-7, 8-dihydroguanine: links to gene expression, aging, and defense against oxidative stress. Free Radic. Biol. Med. 49: 587-596.

Rowe LA, Degtyareva N and Doetsch PW (2008). DNA damage-induced reactive oxygen species (ROS) stress response in Saccharomyces cerevisiae. Free Radic. Biol. Med. 45: 1167-1177.

Silva CR, Monteiro MR, Rocha HM, Ribeiro AF, et al. (2008). Assessment of antimutagenic and genotoxic potential of senna (Cassia angustifolia Vahl.) aqueous extract using in vitro assays. Toxicol. In Vitro 22: 212-218.

Singh KK, Sigala B, Sikder HA and Schwimmer C (2001). Inactivation of Saccharomyces cerevisiae OGG1 DNA repair gene leads to an increased frequency of mitochondrial mutants. Nucleic Acids Res. 29: 1381-1388.

Thomas D, Scot AD, Barbey R, Padula M, et al. (1997). Inactivation of OGG1 increases the incidence of G . C $\rightarrow$ T . A transversions in Saccharomyces cerevisiae: evidence for endogenous oxidative damage to DNA in eukaryotic cells. Mol. Gen. Genet. 254: 171-178.

van Gorkom BA, de Vries EG, Karrenbeld A and Kleibeuker JH (1999). Review article: anthranoid laxatives and their potential carcinogenic effects. Aliment. Pharmacol. Ther. 13: 443-452. 